\title{
Nitrogenous Contamination in Iranian Vegetables: a Review
}

\author{
Ali Almasi', Mitra Mohammadi ${ }^{1 *}$, Abdollah Dargahi', Farhad Amirian², \\ Zeinab Jafari Motlagh ${ }^{1}$, Ghobad Ahmadidoust ${ }^{3}$, Monireh Noori ${ }^{4}$ \\ ${ }^{1}$ Department of Environmental Health Engineering, School of Public Health, Social Development and Health Promotion \\ Research Center, Kermanshah University of Medical Sciences, Kermanshah, Iran \\ ${ }^{2}$ Department of Pathology, School of Medicine, Kermanshah University of Medical Sciences, Kermanshah, Iran \\ ${ }^{3}$ Department of Environmental Health Engineering, School of Public Health, Hamadan University of Medical Sciences, \\ Hamadan, Iran \\ ${ }^{4}$ Student Research Committee, Kermanshah University of Medical Sciences, Kermanshah, Iran
}

Received: 10 October 2017

Accepted: 7 December 2017

\begin{abstract}
Vegetables are the main source of nitrate food contamination. More than $80 \%$ of nitrates are consumed by humans through vegetables. The present study was conducted to investigate the contamination of nitrite and nitrate vegetables in Iran. In this systematic overview we reviewed all internal and external databases for relevant articles. According to different studies, contamination was detected in different types of vegetables in different regions of Iran. Articles that examined nitrite values above the standard limit were not reported. Due to nitrate contamination in vegetables in some cities of Iran and the effects of this nitrogen combination on human health, it is necessary to find a solution in this regard.
\end{abstract}

Keywords: nitrite, nitrate, vegetables, Iran

\section{Introduction}

With increasing population growth in Iran, demand for food is increasing. For this reason, the use of organic and chemical fertilizers is very common in terms of production per unit area [1]. Nitrogen is the most consumed element of the plant that is used in agriculture in large quantities. By increasing nitrogen concentrations in soil and water, nitrogen content in plant tissues increases and nitrate levels are usually associated with the concentration of nitrogen in the plant [2]. The accumulation of nitrate in plants is a natural phenomenon

*e-mail: m.mohamadi725@gmail.com and occurs when accumulation of nitrate in the plant is more than its reduction due to absorption [3]. Vegetables are the main source of food contamination with nitrate, so that they make 300 to $940 \mathrm{mg} / \mathrm{g}$ of daily intake [4]. More than $80 \%$ of human nitrate is fed through vegetables [5]. Nitrate accumulated in vegetables is converted to nitrite and nitros acids and if they are combined with the firstand second-type amines, nitrosamines are produced and their health risks have been proven [6]. Nitrite and nitrate are stored in different vegetables organs. The amount of nitrate accumulation in plants depends on factors such as cultivation, plant variety, age of the plant, climatic conditions, type and amount of fertilization cropping, harvesting time, and how to store the crop after harvest [7]. At high temperatures the ratio of nitrate reduction in 
the root also increases. High levels of nitrate are found in contaminated food or in fragile tissues of vegetables stored for a few days at room temperature [4]. Inappropriate, unbalanced, and excessive use of nitrogen fertilizers in Iran has increased the concentration of nitrate in soil, water, and plants. Also, the application of nitrogen fertilizers is very high due to the effect of nitrogen on plant growth [8]. In humans, nitrate is rapidly absorbed from the stomach and the intestine and at least $25 \%$ is transmitted to the saliva. The concentration in saliva is 10 times that of plasma. In young people, $5-7 \%$ total nitrogen converts to nitrite. In kids and those with stomach ailments because of lower gastric $\mathrm{pH}$, the conversion rate to nitrite is higher [9]. The presence of nitrate and nitrite in food is associated with an increased risk of gastrointestinal cancers in adults and methemoglobinemia in babies. The amount of methemoglobin in the body is usually $1-3 \%$, but more than 10 percent is clinically significant [10]. The lethal dose of nitrate produced by food is $330 \mathrm{mg} / \mathrm{kg}$ body weight and nitrite is 10 times more toxic than nitrate [11]. The United States Environmental Protection Agency (EPA) reported a reference nitrate value of $1.6 \mathrm{mg}$ nitrogen per $\mathrm{kg}$ body weight per day (equivalent to $7 \mathrm{mg}$ nitrate per $\mathrm{kg}$ body weight per day) [4]. In the Joint Expert Committee of the Food and Agriculture (JECFA) and the European Commission's Scientific Committee on Food (SCF), the amount of ADI for nitrate is 0-3.7 for each kilogram of body weight [12]. The consumption of only 100 grams of raw vegetables with a concentration of $2,500 \mathrm{mg} / \mathrm{kg}$ of nitrate leads to the absorption of $250 \mathrm{mg}$ of nitrate. the consumption of this amount of vegetable alone for a person of $60 \mathrm{~kg}$ makes the amount of nitrate absorbed 13\% more than ADI. By calculating the conversion of $5 \%$ of this nitrate to nitrite content after each use, the SCF and ADI levels for nitrite $(0.6 \mathrm{mg} / \mathrm{kg}$ body weight) increased by more than $247 \%$ [4].

The review study of Croitoru et al. (2015) showed that vegetables with the lowest nitrate concentration included lettuce, parsley root, radish, and licorice root, and no correlation was found between nitrite and nitrate levels [13]. Suh et al. in South Korea (2013) found that nitrite and nitrate concentrations in 25 types of vegetable were $0.6-719 \mathrm{mg} / \mathrm{kg}$ and $0.1-635 \mathrm{mg} / \mathrm{kg}$, so that there was no risk to consumers [14]. Considering the different studies in the field of contamination of vegetables with nitrate and nitrite, a critical review of these articles seems necessary in order to obtain a general view of contamination of vegetables used in this dangerous substance in Iran. This study tries to present a comprehensive overview of vegetable contamination by presenting the results of research carried out in this field and suggests ways to reduce nitrate contamination.

\section{Materials and Methods}

Iran's $1,648,195 \mathrm{~km}^{2}$ make it the $18^{\text {th }}$ largest country by area in the world, and based on the census of 2016 has $79,926,270$ people [15]. Iran is semi-arid and dry in terms of precipitation. In agriculture and horticulture Iran has high rankings for some products. According to 2007 statistics, the country is 2 nd in world for cucumber production, and 4th and 5th for melon and watermelon, respectively [16]. In this study, nitrite and nitrate contamination in vegetables were surveyed in different cities of Iran.

\section{Result and Discussion}

The results of the study on nitrite and nitrate of vegetables are presented in Tables 1-3. In some areas based on the culture and habits of people in the cities and the fertile nature of some areas due to the geographical location, cultivating a variety of vegetables takes place. The results of the study indicate that nitrogen (nitrite and nitrate) compounds are present in all of the studied products in Iranian cities. Therefore, in order to clarify the control of nitrate and nitrite in vegetables grown in Iran's cities it is necessary to pay enough attention from the authorities and farmers on the proper fertilization and irrigation of vegetables in order to develop optimum products with low nitrate content. First of all, it is necessary to explain that the standard nitrate in $\mathrm{mg} / \mathrm{kg}$ in cabbage, lettuce, spinach, radish, carrots, tomato, potato, celery, onions, and eggplants is respectively 5,000, 3,000, $3,500,1,500,1,400,300,150,60,5,000,1,000$, and 2,000. In the study of the effect of traditional and mechanized crops on the level of contamination of tomato and potato in Khorasan Razavi [30], we determined that the culture did not have a significant effect on potato contamination and in both types of culture, and the amount of nitrate was less than standard. But the amount of tomato contamination in mechanized crops (18.3 times the standard limit) was higher than traditional culture (3.41 times the standard limit), and this difference was statistically significant $(\mathrm{P}<0.05)$. In a study conducted in Isfahan, the effect of irrigation water on nitrate and nitrate accumulation in vegetables was studied [38]. In this study, nitrate levels in tomato, cucumber, onion, lettuce, and spinach in both irrigation methods were found to be lower than the standard limit. However, nitrate content in irrigated potatoes with Zayandeh Rood water (1.07 times the limit) was lower than that observed in well water wells (1.94) in irrigated potatoes, and this relationship was significant $(\mathrm{P}<0.05)$. According to various reports from different cities of Iran, the nitrate content in carrots in Isfahan and Shiraz was exceeded and permitted in Ahvaz [30-31, 35].

In the Nuezde Gonzlez et al. (2015) study on nitrate and nitrate in raw vegetables in the United States, 124 fresh vegetable types such as cabbage, lettuce, and spinach were examined as organic and conventional cultures from 5 cities located in different geographic regions. There was no difference in the average concentration of these 2 cultivars. But the amount of nitrate was significant. The concentration of nitrate in organic cultures was lower [43]. The study of Iammarino et al. (2014) on 
Table 1. Nitrite and nitrate $(\mathrm{mg} / \mathrm{kg})$ in different types of vegetables supplied in west, east, north, and south of Iran.

\begin{tabular}{|c|c|c|c|c|c|}
\hline \multicolumn{6}{|c|}{ West of Iran } \\
\hline Reference & City & Type of vegetable & Nitrate, $\mathrm{mg} / \mathrm{kg}$ & Nitrite, $\mathrm{mg} / \mathrm{kg}$ & Others note \\
\hline \multirow{9}{*}{$\begin{array}{l}\text { Pirsaheb et } \\
\text { al. }(2010) \\
\quad[15]\end{array}$} & \multirow{9}{*}{ Kemanshah } & Radish leaves & 0.4 & - & \multirow{9}{*}{$\begin{array}{l}\text { Mean concentration of nitrate in potato } \\
\text { was higher than standard level. Nitrat } \\
\text { content depend on use of nitrate ferti- } \\
\text { lizer, season, type of product is different. }\end{array}$} \\
\hline & & Radish & 4.3 & - & \\
\hline & & Cabbage & 0.9 & - & \\
\hline & & Lettuce & 0.4 & - & \\
\hline & & Celery & 1.2 & - & \\
\hline & & Potato & 0.69 & - & \\
\hline & & Tomato & 3 & - & \\
\hline & & Melon & 0.6 & - & \\
\hline & & Watermelon & 0.58 & - & \\
\hline \multirow{14}{*}{$\begin{array}{l}\text { Pirsaheb et } \\
\text { al. (2011) } \\
\quad[16]\end{array}$} & \multirow{5}{*}{$\begin{array}{l}\text { Dorood fara- } \\
\text { man }\end{array}$} & Mint & 284.09 & 0.28 & \multirow{14}{*}{$\begin{array}{l}\text { Unlike nitrate, mean different of nitrite } \\
\text { content in all samples was significant } \\
(\mathrm{p}<0.05) \text {. Maximum and minimum of } \\
\text { nitrite obtained in leek and cucumber, } \\
\text { respectively. }\end{array}$} \\
\hline & & Melon & 91.1 & 0.1 & \\
\hline & & Watermelon & 31.65 & 0.356 & \\
\hline & & Cucumber & - & 0.17 & \\
\hline & & Tomato & - & 0.17 & \\
\hline & \multirow{9}{*}{ Mahidasht } & Mint & - & 0.59 & \\
\hline & & Spinach & - & 1.58 & \\
\hline & & Radish & - & 6.05 & \\
\hline & & Radish leave & - & 0.63 & \\
\hline & & Leeks & - & 1.78 & \\
\hline & & Onion & - & 0.36 & \\
\hline & & Melon & - & 0.13 & \\
\hline & & Watermelon & - & 0.1 & \\
\hline & & Cucumber & - & 0.1 & \\
\hline \multirow{4}{*}{$\begin{array}{l}\text { Shahabza- } \\
\text { degan et al. } \\
(2010)[17]\end{array}$} & \multirow{4}{*}{ Ardebil } & Onion leaves & 1555.8 & - & \multirow{4}{*}{$\begin{array}{l}\text { Nitrate concentrations in leafy vegetables } \\
\text { and root tuber is more than vegetables. } \\
\text { Fruits than vegetables nitrate levels are } \\
\text { low. The highest nitrate concentrations } \\
\text { at spring onion, purple, cabbage, spinach } \\
\text { leaves and red apples and yellow were } \\
\text { the lowest level. }\end{array}$} \\
\hline & & Purple cabbage & 1394.8 & - & \\
\hline & & Spinach & 1021 & - & \\
\hline & & Tomato & - & 0.12 & \\
\hline \multirow{11}{*}{$\begin{array}{l}\text { Nouwrouz } \\
\text { et al. }(2012) \\
\quad[18]\end{array}$} & \multirow{11}{*}{ Tabriz } & Cabbage & 161 & - & \multirow{11}{*}{$\begin{array}{l}\text { Nitrate concentrations were below of } \\
\text { others reported at different countries. } \\
\text { The mean concentration of nitrate at all } \\
\text { vegetables in autumn was higher than in } \\
\text { spring significantly. }\end{array}$} \\
\hline & & Lettuce & 781 & - & \\
\hline & & Spinach & 83 & - & \\
\hline & & Parsley & 707 & - & \\
\hline & & Coriander & 441 & - & \\
\hline & & Dill & 501 & - & \\
\hline & & Leeks & 1702 & - & \\
\hline & & Fenugreek & 684 & - & \\
\hline & & Tarragon & 805 & - & \\
\hline & & Fumitory & 772 & - & \\
\hline & & Mint & 191 & - & \\
\hline
\end{tabular}


Table 1. Continued.

\begin{tabular}{|c|c|c|c|c|c|}
\hline \multicolumn{6}{|c|}{ West of Iran } \\
\hline Reference & City & Type of vegetable & Nitrate, $\mathrm{mg} / \mathrm{kg}$ & Nitrite, $\mathrm{mg} / \mathrm{kg}$ & Others note \\
\hline $\begin{array}{l}\text { Sadeghi et } \\
\text { al. (2013) } \\
\quad[19]\end{array}$ & Kermanshah & Coriander & 128.17 & 115.32 & $\begin{array}{l}\text { The concentrations of nitrate and nitrite } \\
\text { were significantly different }(\mathrm{p}<0.5) \text { in } \\
\text { terms of vegetable type. vegetables. It is } \\
\text { crucial to monitor and control the quality } \\
\text { of this product and studying other food } \\
\text { processing because of the daily intake of } \\
\text { vegetables and potential risks of nitrate } \\
\text { and nitrite accumulation and itsassocia- } \\
\text { tion with some illnessesand gastrointesti- } \\
\text { nal tract cancers. }\end{array}$ \\
\hline \multirow{5}{*}{$\begin{array}{l}\text { Rostamzade } \\
\text { at al. (2013) } \\
\text { [20] }\end{array}$} & \multirow{5}{*}{ Tabriz } & Cucumber & $1800-1900$ & - & \multirow{5}{*}{$\begin{array}{l}\text { Use of nitrogen fertilizer in cucumber } \\
\text { was high. }\end{array}$} \\
\hline & & Parsley & 214.33 & 123.33 & \\
\hline & & Torre & 235 & 371.7 & \\
\hline & & Spinach & 103.33 & 99.17 & \\
\hline & & Scallion & 49.17 & 42.5 & \\
\hline \multirow{5}{*}{$\begin{array}{l}\text { Rezaeei et } \\
\text { al. (2014) } \\
\quad[21]\end{array}$} & \multirow{5}{*}{ Arak } & Cucumber & 42.7 & 9.03 & \multirow{5}{*}{$\begin{array}{c}\text { Mean of nitrate concentration was lower } \\
\text { than WHO standard. Nitrite concentra- } \\
\text { tion in some samples was higher than } \\
\text { standard. }\end{array}$} \\
\hline & & Tomato & 7.82 & 1.81 & \\
\hline & & Watermelon & 26.61 & 5.5 & \\
\hline & & Cantaloupe & 58.98 & 28.36 & \\
\hline & & Melon & 33.64 & 7.65 & \\
\hline $\begin{array}{l}\text { Pirsaheb et } \\
\text { al. (2014) } \\
{[22]}\end{array}$ & Kermanshah & Tomato & 0.65 & 10.56 & $\begin{array}{l}\text { Different of nitrate concentration in } \\
\text { south of Mian Darband region was } \\
\text { higher than others. }\end{array}$ \\
\hline \multirow{5}{*}{$\begin{array}{l}\text { Eftekhari et } \\
\text { al. (2014) } \\
\text { [23] }\end{array}$} & Tabriz & \multirow{5}{*}{ Spinach } & 5151 & 112.1 & \\
\hline & Oromie & & 5692.5 & 104.2 & \\
\hline & Zanjan & & 6775.5 & 132.7 & \\
\hline & Khoramabad & & 5889.8 & 93.95 & \\
\hline & Hamedan & & 5692.5 & 187.8 & \\
\hline \multirow{2}{*}{$\begin{array}{l}\text { Lahiji et al. } \\
\quad[24]\end{array}$} & \multirow{2}{*}{ Hamedan } & \multirow{2}{*}{ Potato } & 1058 & - & \multirow{2}{*}{$\begin{array}{l}\text { Nitrate residual was higher than Iran } \\
\text { standard level. }\end{array}$} \\
\hline & & & 1000 & - & \\
\hline \multirow{3}{*}{$\begin{array}{l}\text { Shariati-Rad } \\
\text { et al. (2016) } \\
\text { [25] }\end{array}$} & \multirow{3}{*}{ Kermanshah } & Lettuce & - & 0.294 & \\
\hline & & Cabbage & - & 0.051 & \\
\hline & & Cucumber & - & ND & \\
\hline \multirow{2}{*}{$\begin{array}{l}\text { Fazlzadeh } \\
\text { et al. (2016) } \\
{[26]}\end{array}$} & \multirow{2}{*}{ Ardabil } & $\begin{array}{l}\text { Potato (chemical } \\
\text { fertilizer) }\end{array}$ & 122.6 & - & \multirow{2}{*}{$\begin{array}{l}\text { using organic fertilizer in agriculture } \\
\text { industries can be considered in long-term } \\
\text { programs of fisheries organizations to } \\
\text { reduce adverse effects. }\end{array}$} \\
\hline & & $\begin{array}{l}\text { Potato (organic } \\
\text { fertilizer ) }\end{array}$ & 85.5 & - & \\
\hline \multirow{2}{*}{$\begin{array}{l}\text { Mousavi } \\
\text { Moayeid et } \\
\text { al. }(2016) \\
{[27]}\end{array}$} & \multirow{2}{*}{ Hamadan } & White Onion & $\begin{array}{l}60.65 \\
\text { (April) } \\
194.85 \text { (Febru- } \\
\text { ary) }\end{array}$ & - & \multirow{2}{*}{$\begin{array}{l}\text { The concentration of nitrate in red onions } \\
\text { of collected in April and white } \\
\text { onions collected in February are more } \\
\text { than WHO standard. the reason of high } \\
\text { nitrate } \\
\text { is probably use of unsuitable, unbalanced } \\
\text { and high amount of nitrate manures. }\end{array}$} \\
\hline & & Red onion & $\begin{array}{c}275.97 \\
\text { (April) } \\
244.67 \\
\text { (February) }\end{array}$ & - & \\
\hline
\end{tabular}


Table 1. Continued.

\begin{tabular}{|c|c|c|c|c|c|}
\hline \multicolumn{6}{|c|}{ East of Iran } \\
\hline \multirow{4}{*}{$\begin{array}{l}\text { Jalini et al. } \\
\text { (2011)[28] }\end{array}$} & $\begin{array}{l}\text { Khorasane } \\
\text { razavi } \\
\text { In Traditional } \\
\text { farms }\end{array}$ & \multirow[t]{2}{*}{ Potato } & 61.7 & - & \multirow{4}{*}{$\begin{array}{l}\text { Use of fertilizers was higher than standard } \\
\text { suggested level. Mean different of nitrite con- } \\
\text { tent in mechanized and conventional methods } \\
\text { and type of product was significant }(\mathrm{p}<0.05 \text { ). }\end{array}$} \\
\hline & $\begin{array}{l}\text { In mechanized } \\
\text { farms }\end{array}$ & & 49.1 & - & \\
\hline & $\begin{array}{l}\text { In Traditional } \\
\text { farms }\end{array}$ & \multirow{2}{*}{ Tomato } & 1024.1 & - & \\
\hline & $\begin{array}{l}\text { In mechanized } \\
\text { farms }\end{array}$ & & 5651.6 & - & \\
\hline \multirow{10}{*}{$\begin{array}{l}\text { Eftekhari et } \\
\text { al. (2014) } \\
\quad[23]\end{array}$} & Shirvan & \multirow{10}{*}{ Spinach } & 6234 & 139.6 & \\
\hline & Ghochan & & 6369 & 132.2 & \\
\hline & Birjand & & 6595.5 & 115 & \\
\hline & Kerman & & 6009 & 127.8 & \\
\hline & Sarasiab & & 5940 & 143.3 & \\
\hline & Mahan & & 3594 & 80.20 & \\
\hline & Ravar & & 5286 & 107.8 & \\
\hline & Zabol & & 4135.5 & 129.8 & \\
\hline & Yazd & & 5286 & 107.8 & \\
\hline & Boroojerd & & 6099 & 101.8 & \\
\hline \multicolumn{6}{|c|}{ North of Iran } \\
\hline \multirow{3}{*}{$\begin{array}{l}\text { Shokrzade } \\
\text { et a.l (2007) } \\
{[29]}\end{array}$} & Ghaemshahr & Leek & 77 & ND & \multirow{3}{*}{$\begin{array}{l}\text { The average of nitrate and nitrite content in } \\
\text { all of the samples was less than standard lim- } \\
\text { it.spinach sampeld from sari had significantly } \\
\text { lower nitrate content than } 2 \text { other cities. }\end{array}$} \\
\hline & Babol & C. & 313 & ND & \\
\hline & Sari & Spritacit & 346 & ND & \\
\hline \multirow{2}{*}{$\begin{array}{l}\text { Eftekhari et } \\
\text { al. }(2014) \\
\text { [23] }\end{array}$} & Sari & \multirow{2}{*}{ Spinach } & 5715.2 & 92.94 & \\
\hline & Tonekabon & & 5106 & 140.06 & \\
\hline \multicolumn{6}{|c|}{ South of Iran } \\
\hline \multirow{14}{*}{$\begin{array}{l}\text { Shahlaei et } \\
\text { al. (2007) } \\
\text { [30] }\end{array}$} & \multirow{14}{*}{ Ahvaz } & Cabbage & $428.3-503.8$ & 4.21 & \multirow{14}{*}{$\begin{array}{l}\text { The highest and lowest nitrate content were } \\
\text { for tomato and mint respectively.in the } \\
\text { almost of the vegetables the nitrate content } \\
\text { was higher than limited values. }\end{array}$} \\
\hline & & Lettuce & $862-583.1$ & 4.16 & \\
\hline & & Spinach & $748-886.5$ & 2.96 & \\
\hline & & radish leave & $179-495.6$ & 3.03 & \\
\hline & & Cress & $434.2-678.5$ & 4.38 & \\
\hline & & Fennel & $313.3-654.1$ & 2.58 & \\
\hline & & Basil & $948.8-996.2$ & 2.56 & \\
\hline & & Mint & $154.5-349.6$ & 1.51 & \\
\hline & & Coriander & $310.1-569.4$ & 2.12 & \\
\hline & & Parsley & $358.6-374$ & 2.23 & \\
\hline & & Carrot & $251.9-458.3$ & 3.29 & \\
\hline & & Pepper & $574.5-622.5$ & 6.96 & \\
\hline & & Celery & $887.4-2169$ & 3.66 & \\
\hline & & Onion & $248.4-1263$ & 4.93 & \\
\hline
\end{tabular}


Table 1. Continued.

\begin{tabular}{|c|c|c|c|c|c|}
\hline \multirow{5}{*}{$\begin{array}{l}\text { Shahlaei et } \\
\text { al. (2007) } \\
{[30]}\end{array}$} & \multirow{5}{*}{ Ahvaz } & Garlic & $197.7-229.3$ & 2.72 & \multirow{5}{*}{$\begin{array}{l}\text { The highest and lowest nitrate content were } \\
\text { for tomato and mint respectively.in the } \\
\text { almost of the vegetables the nitrate content } \\
\text { was higher than limited values. }\end{array}$} \\
\hline & & Tomato & $1644-1681$ & 9.52 & \\
\hline & & Cucumber & $813.3-999.3$ & 4.97 & \\
\hline & & Potato & $155-213.8$ & 1.83 & \\
\hline & & Eggplant & $652.4-864.5$ & 6.94 & \\
\hline \multirow{5}{*}{$\begin{array}{l}\text { Rousta et al. } \\
(2010)[31]\end{array}$} & \multirow{5}{*}{ Shiraz } & Carrots & 4641 & - & \multirow{5}{*}{$\begin{array}{l}\text { Nitrate level in more vegetable was higher } \\
\text { than standard. }\end{array}$} \\
\hline & & Lettuce & 105 & - & \\
\hline & & Cucumber & 673 & - & \\
\hline & & Tomato & 942 & - & \\
\hline & & Potato & 657 & - & \\
\hline \multirow{26}{*}{$\begin{array}{l}\text { Afali et al. } \\
\text { (2014) [32] }\end{array}$} & \multirow{26}{*}{ Shiraz } & Carrot & 60.60 & 8.50 & \multirow{26}{*}{$\begin{array}{l}\text { Nitrate concentration in east of shiraz was } \\
\text { higher than others. That may be in relation to } \\
\text { harvest time, season, Vegetation, save time. }\end{array}$} \\
\hline & & Turnip & 37.80 & 6.45 & \\
\hline & & Onion & 36.9 & 6.09 & \\
\hline & & Green beans & 50.88 & 7.64 & \\
\hline & & Pepper & 42.6 & 6.56 & \\
\hline & & Torre & 74.4 & 6.75 & \\
\hline & & Savory & 73.2 & 9.9 & \\
\hline & & Celery & 46.08 & 6.3 & \\
\hline & & Mint & 74.8 & 8.7 & \\
\hline & & Parsley & 46.4 & 8.78 & \\
\hline & & Lettuce & 54.7 & 7.8 & \\
\hline & & Spinach & 44 & 6.6 & \\
\hline & & Dill & 54 & 7.4 & \\
\hline & & Basil & 55.8 & 8.8 & \\
\hline & & Tarragon & 50.4 & 8.4 & \\
\hline & & Leeks & 37.2 & 7.7 & \\
\hline & & cabbage & 56.4 & 6.9 & \\
\hline & & Purple cabbage & 48.2 & 7.65 & \\
\hline & & Eggplant & 66.93 & 8.63 & \\
\hline & & Potato & 73.40 & 8.1 & \\
\hline & & Cucumber & 56.066 & 6.84 & \\
\hline & & Tomato & 33.6 & 5.6 & \\
\hline & & Tomato & 7.82 & 1.81 & \\
\hline & & Watermelon & 26.61 & 5.5 & \\
\hline & & Cantaloupe & 58.98 & 28.36 & \\
\hline & & Melon & 33.64 & 7.65 & \\
\hline $\begin{array}{l}\text { Keshavarz } \\
\text { et al. (2015) } \\
{[33]}\end{array}$ & Shiraz & Spinach & 336.54 & 26.49 & $\begin{array}{l}\text { HPLC method was used to measure the } \\
\text { nitrite and nitrate levels. }\end{array}$ \\
\hline
\end{tabular}


Table 1. Continued.

\begin{tabular}{|c|c|c|c|c|c|}
\hline \multirow[b]{2}{*}{$\begin{array}{c}\text { Gholami et } \\
\text { al. (2013) } \\
{[34]}\end{array}$} & \multirow[b]{2}{*}{ Dezfol } & Coriander & 2317 & - & \multirow{2}{*}{$\begin{array}{l}\text { Results of analyses indicated that the highest } \\
\text { amount of nitrate was seen in the samples } \\
\text { from north of Dezful. . Moreover, nitrate } \\
\text { concentration was reported more than per- } \\
\text { mitted in coriander. As well, nitrate concen- } \\
\text { tration tested in the morning and afternoon } \\
\text { samples showed significant difference and } \\
\text { the highest difference was related to north } \\
\text { and western of Dezful. }\end{array}$} \\
\hline & & Torre & 1474 & - & \\
\hline \multirow{2}{*}{$\begin{array}{c}\text { Eftekhari et } \\
\text { al. (2014) } \\
{[23]}\end{array}$} & Fasa & \multirow{2}{*}{ Spinach } & 3393 & 96.36 & \\
\hline & Shiraz & & 6211.5 & 125.3 & \\
\hline
\end{tabular}

Table 2. Nitrite and nitrate $(\mathrm{mg} / \mathrm{kg})$ in different types of vegetables supplied in centre of Iran.

\begin{tabular}{|c|c|c|c|c|c|}
\hline Reference & City & Type of vegetable & Nitrate, $\mathrm{mg} / \mathrm{kg}$ & $\begin{array}{l}\text { Nitrite, } \\
\mathrm{mg} / \mathrm{kg}\end{array}$ & Others note \\
\hline \multirow{11}{*}{$\begin{array}{l}\text { Sobhan et al . } \\
\text { (2010) [35] }\end{array}$} & \multirow{11}{*}{ Esfahan } & Spinach & $1620-47235$ & - & \multirow{11}{*}{$\begin{array}{c}\text { Maximum and minimum nitrate } \\
\text { concentration In leafy greens was dill } \\
(2120.9 \mathrm{mg} / \mathrm{kg}) \text { and spinach }(20.7 \mathrm{mg} / \mathrm{kg}) \\
\text { Onion }(237.7 \mathrm{mg} / \mathrm{kg}) \text { and cucumber } \\
(89 \mathrm{mg} / \mathrm{kg}) \text { were maximum and minimum } \\
\text { of nitrate in vegetables glandular. }\end{array}$} \\
\hline & & Basil & $5513-7695$ & - & \\
\hline & & onion & $2135-8440$ & - & \\
\hline & & Fenugreek & $909-15800$ & - & \\
\hline & & Leeks & $2390-5590$ & - & \\
\hline & & Cabbage & $3650-5550$ & - & \\
\hline & & Lettuce & $2210-3640$ & - & \\
\hline & & carrot & $982-12700$ & - & \\
\hline & & Tomato & $104-152$ & - & \\
\hline & & Potato & $701-4450$ & - & \\
\hline & & Cucumbers & $255-330$ & - & \\
\hline \multirow{15}{*}{$\begin{array}{c}\text { Kafeshani } \\
\text { et al. (2013) } \\
{[36]}\end{array}$} & \multirow{7}{*}{$\begin{array}{l}\text { Esfahan (Zay- } \\
\text { andeh rood) }\end{array}$} & $\begin{array}{l}\text { Leeks } \\
\text { Lettuce }\end{array}$ & $\begin{array}{c}230.66 \\
173.8\end{array}$ & $\begin{array}{l}\text { ND } \\
\text { ND }\end{array}$ & \multirow{15}{*}{$\begin{array}{l}\text { Mean different of nitrate in spinach and } \\
\text { parsley was significant. }\end{array}$} \\
\hline & & Parsley & 319 & ND & \\
\hline & & Spinach & 321.25 & ND & \\
\hline & & Onion & 45.25 & ND & \\
\hline & & Cucumbers & 30 & ND & \\
\hline & & Tomato & 30.66 & ND & \\
\hline & & Potato & 64.25 & ND & \\
\hline & \multirow{8}{*}{$\begin{array}{c}\text { Esfahan } \\
\text { (Well water) }\end{array}$} & Leeks & 161.83 & ND & \\
\hline & & Lettuce & 275.71 & ND & \\
\hline & & Parsley & 312.5 & ND & \\
\hline & & Spinach & 311 & ND & \\
\hline & & Onion & 28.41 & ND & \\
\hline & & Cucumbers & 26.73 & ND & \\
\hline & & Tomato & 26.66 & ND & \\
\hline & & Potato & 116.9 & ND & \\
\hline
\end{tabular}


Table 2. Continued.

\begin{tabular}{|c|c|c|c|c|c|}
\hline Reference & City & Type of vegetable & Nitrate, $\mathrm{mg} / \mathrm{kg}$ & $\begin{array}{l}\text { Nitrite, } \\
\mathrm{mg} / \mathrm{kg}\end{array}$ & Others note \\
\hline \multirow{10}{*}{$\begin{array}{c}\text { Rahmani et } \\
\text { al. (2013) } \\
{[37]}\end{array}$} & \multirow{10}{*}{ Esfahan } & Dill & $989.8-6297.9$ & - & \multirow{10}{*}{$\begin{array}{c}\text { The concentration of nitrates in leafy vegeta- } \\
\text { bles to herbs was more glandular. In general, } \\
\text { leafy vegetables (except spinach) and herbs } \\
\text { gland (except cucumbers) had nitrate con- } \\
\text { centrations higher than usual nitrate in the } \\
\text { plant. }\end{array}$} \\
\hline & & Mint & 872.4-3386.1 & - & \\
\hline & & Fenugreek & $821.9-3400.3$ & - & \\
\hline & & Chard & $1555.3-2210.3$ & - & \\
\hline & & Coriander & 1068.6-1759.0 & - & \\
\hline & & Parsley & 914.9-2181.15 & - & \\
\hline & & Torre & $694.0-2181.5$ & - & \\
\hline & & Onion & $239.1-872.4$ & - & \\
\hline & & Tomato & $17.7-296.7$ & & \\
\hline & & Potato & $105.4-204.6$ & & \\
\hline $\begin{array}{c}\text { Saeedifar et } \\
\text { al. (2014) } \\
{[38]}\end{array}$ & Tehran & Eggplant & 279.76 & - & $\begin{array}{l}\text { Nitrate level in south of Tehran was higher } \\
\text { than others zones. }\end{array}$ \\
\hline \multirow{4}{*}{$\begin{array}{c}\text { Bahadoran } \\
\text { et al. (2016) } \\
{[39]}\end{array}$} & \multirow{4}{*}{ Tehran } & Leafy vegetables & 276.53 & 5.13 & \multirow{4}{*}{$\begin{array}{l}\text { Vegetables with the highest nitrate concen- } \\
\text { trations (mg } 100 \text { g_1) included radish (625), } \\
\text { beetroot (495), tarragon (424), lettuce (365), } \\
\text { mint (279), and celery (261). nitrate/nitrite } \\
\text { contents of some vegetables were higher } \\
\text { than the previous report }\end{array}$} \\
\hline & & Roots & 348.57 & 4.77 & \\
\hline & & Starchy vegetables & 51.23 & 5.13 & \\
\hline & & Other vegetables & 88.54 & 4.82 & \\
\hline \multirow{23}{*}{$\begin{array}{c}\text { Pormoghim } \\
\text { et a.l }(2010) \\
{[40]}\end{array}$} & \multirow{3}{*}{ Tehran } & Lettuce & 1123.46 & - & \multirow{23}{*}{$\begin{array}{l}\text { Order of nitrate concentration was leafy } \\
\text { greens }>\text { vegetables glandular }>\text { Vegetable } \\
\text { plant.Nitrate level in lettuce was according } \\
\text { to standard and its mean different of nitrate } \\
\text { content with Potato and Tomato was signifi- } \\
\text { cant }(\mathrm{p}<0.05) \text {. }\end{array}$} \\
\hline & & Potato & 155.65 & - & \\
\hline & & Tomato & 188.41 & - & \\
\hline & Khorasegan & Spinach & 9325 & - & \\
\hline & ziar & \multirow{2}{*}{ Leeks } & 5410 & - & \\
\hline & hanshoie & & 4710 & - & \\
\hline & khorasegan & \multirow{4}{*}{ Basil } & 7695 & - & \\
\hline & dastgerd & & 5513 & - & \\
\hline & Yaraan & & 15600 & - & \\
\hline & Jarghoie & & 7370 & - & \\
\hline & Ziar & \multirow{8}{*}{ Fenugreek } & 909 & - & \\
\hline & Ghriden & & 15800 & - & \\
\hline & Gort & & 1925 & - & \\
\hline & Varsian & & 3590 & - & \\
\hline & Ayad in & & 5420 & - & \\
\hline & Asfina & & 2440 & - & \\
\hline & Abad asghar & & 4700 & - & \\
\hline & $\begin{array}{l}\text { Bagh paran- } \\
\text { degan }\end{array}$ & & 3730 & - & \\
\hline & Jozdan & \multirow{5}{*}{ Onion } & 4960 & - & \\
\hline & Khorasegan & & 4600 & - & \\
\hline & Dashti & & 2135 & - & \\
\hline & Darche & & 2320 & - & \\
\hline & Felavarjan & & 8440 & - & \\
\hline
\end{tabular}


Table 2. Continued.

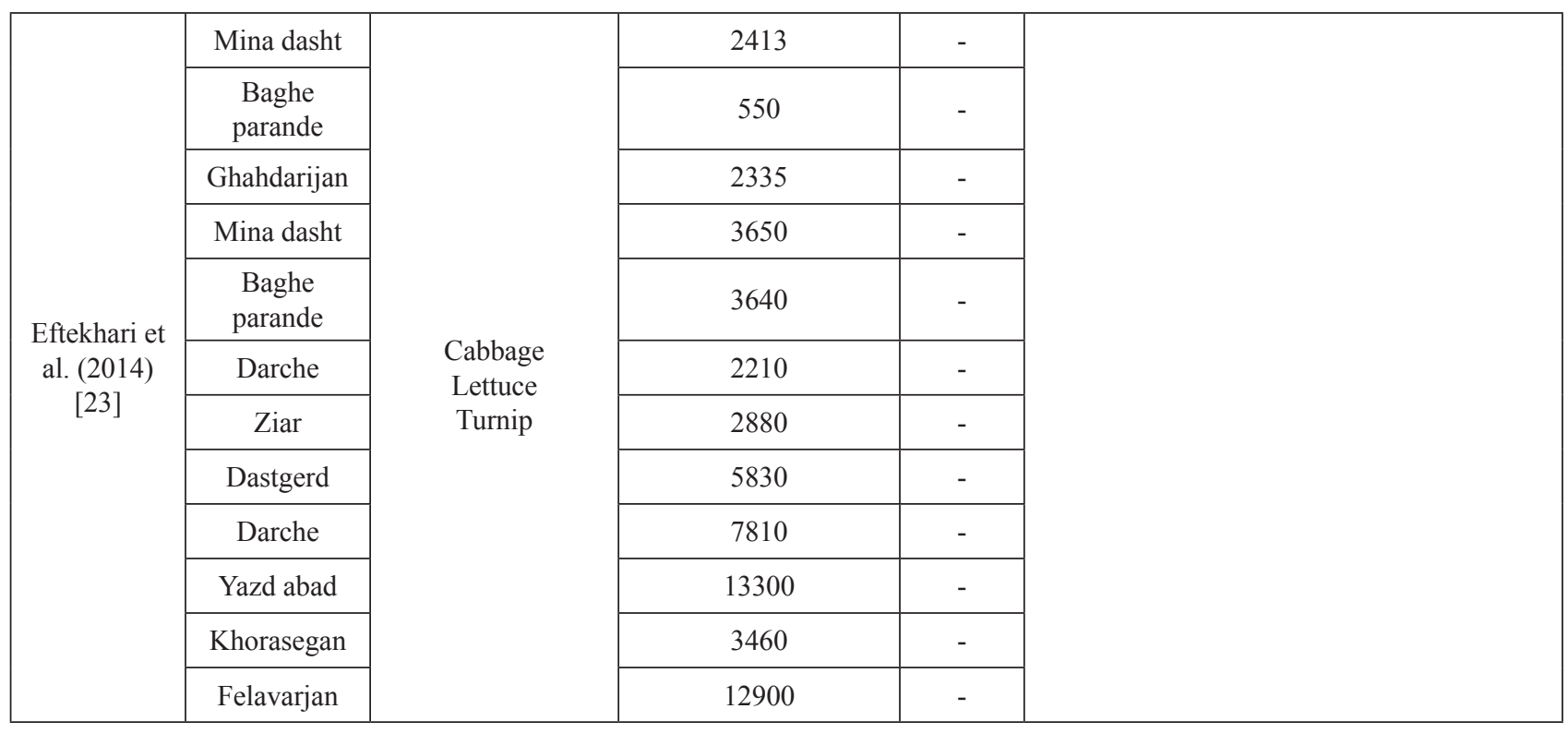

nitrite and nitrate of 150 leafy vegetables and their risk assessment showed that nitrate in spinach and lettuce is higher than the standard limit. Nitrite levels also varied from 28.5 to $19.5 / 19$ [44]. Investigating studies on lettuce and eggplant in Iran, nitrate pollution of these products was not exceeded by standards [16, 30, 35-36, 38, 40]. Also, the amount of nitrate in potatoes in most cities was higher than the limit, so that Isfahan had the highest rate of pollution among the cities of Iran with 74.1-11.68-fold [37].
Spinach is highly regarded by farmers and nutritionists due to its resistance to contamination rooting rapid growth and nativeness. Spinach nitrate contamination has been reported in most Iranian cities [18, 35]. So that, the lowest and the highest amounts of rape exceeded the standard limit in coronans was 46.45 and 0.05 times in Varamin. However, the results of studies in Kermanshah [1], Isfahan [36], Fasa [23], Sari, Ghaemshahr and Babol [29], Ahvaz [30], Shiraz [32], and Ardabil [17] do not indicate any nitrate contamination of spinach. It should be noted

Table 3. Range of nitrogen contamination in different cities of Iran.

\begin{tabular}{|c|c|c|c|c|}
\hline \multirow{2}{*}{ Type of vegetable } & \multicolumn{2}{|c|}{ Nitrate concentration $(\mathrm{mg} / \mathrm{kg})$} & \multicolumn{2}{|c|}{ Nitrite concentration $(\mathrm{mg} / \mathrm{kg})$} \\
\hline & Minimum (city) & Maximum (city) & Minimum (city) & Maximum (city) \\
\hline Lettuce & 54.7 (Shiraz) & 2210-3460 (Esfahan) & 4 (Ahvaz) & 7.8 (Shiraz) \\
\hline Tomato & 0.32 (Kermanshah) & 5651.6 (Khorasan razavi) & 0.125 (Mahidasht) & 11.19 (Kermanshah) \\
\hline Carrot & 60.6 (Shiraz) & 982-12700 (Esfahan) & - & - \\
\hline Spinach & 83 (Tabriz) & 1620-47235 (Esfahan) & 6.6(Shiraz) & 99.17(Kermanshah) \\
\hline Leek & 191 (Tabriz) & 4710 (Hanshoyeh) & - & - \\
\hline Potato & 2.43 (Tabriz) & 701-4450 (Esfahan) & - & - \\
\hline Onion & 28.4 (Esfahan) & 7335 (Ziar) & 0.25 (Dorood Faraman) & 6.6(Shiraz) \\
\hline Cucumber & 26.73 (Esfahan) & 999 (Ahvaz) & 0.1 (Mahidasht) & 5.3 (Ahvaz) \\
\hline Water melon & 0.58 (Kermanshah) & 31.65 (Mahidasht) & - & - \\
\hline Melon & 0.6 (Kermanshah) & 91.1 (Mahidasht) & - & - \\
\hline Mint & 74.8(Shiraz) & 872.4-3386.1 (Esfahan) & 1.13 (Ahvaz) & 8.7 (Shiraz) \\
\hline Coriander & 115 (Kermanshah) & 1068.6-1759(Esfahan) & - & - \\
\hline Celery & 1.2(Kermanshah) & 46.08 (Shiraz) & - & - \\
\hline Eggplant & 66.93(Shiraz) & 864 (Ahvaz) & - & - \\
\hline Cabbage & 48.2(Shiraz) & 3650-5550 (Esfahan) & - & - \\
\hline
\end{tabular}


that the level of pollution to nitrite exceeds the standard limit in any of the studied products in Iranian cities. In the study of celery greens in Kermanshah and Ahvaz, nitrate pollution was found to be lower than the limit [16, 30]. While the amounts of nitrate pollution of cabbage vegetables in Minadasht, Isfahan, and Ardebil were 4.67, 11.1-7.3, and 2.8 times more than standards, respectively, and in Shiraz and Tabriz were less than standard [17, 18, $32-33,35]$. In this study, tomato was a product that was less polluted by nitrogen fertilizers and only in Khorasan Razavi was nitrate content 18.3 times higher than the standard [28]. The results of these studies confirmed the high nitrate content in leafy vegetables compared to the other group of vegetables, which had the highest nitrate in radish and celery leaves, as well as high nitrite concentrations in tomato and radish tubers and low levels of it in lettuce. Crude spinach with low nitrate content but high nitrite content showed that this could be due to the weather conditions, the amount of fertilizer used, and other conditions of growth. Nitrate concentrations in leafy vegetables were higher than those of the tuber and in the tuber vegetables more than the fruits $[29,36,38,40]$. In general, the nitrate enters the body through pepper, mint, potatoes, and eggplant, and the minimum nitrate is devoted to savory vegetables such as onions and tomato [32]. Nitrate in leafy vegetables of Ahvaz was lower than the standard level. So these vegetables are harmless and can be consumed. In Kermanshah [22] the average nitrate in all tomato samples from Mian Darband was lower than the standard. Therefore, these products have a virtual nitrate level for various applications such as tomato paste production. The results of the measurements also showed that the rate of nitrogen fertilizers in most farms was more than the fertilizer recommendation. Excessive use of nitrogen, in addition to reducing yield, can increase nitrate concentrations in vegetables with excessive concentrations of undesirable properties for this product. In addition, excessive use of nitrogen fertilizers may increase part of it in the form of nitrate, which has been a long-term threat to human health. According to studies, a high level of nitrite and nitrate in the diet is known to be a cause of gastric cancer.

The existence of high nitrite and nitrate in some products has been observed [45]. The nitrate average in potatoes distributed in Kermanshah was more than acceptable. High levels of nitrate can be attributed to the high consumption of nitrogen fertilizers, agronomic factors, the mechanization of agriculture, genetic sagriculture (e.g. soil type, dosage, and chemical forms of nitrogen availability of other nutrients, herbicide, etc.) and moisture [46]. Proper management practices such as the right manure selection seek soil testing and the elimination of subsidies for chemical fertilizers and the use of organic materials and biological fertilizers in order to reduce the risk of nitrate accumulation and the safe keeping of manufactured products [31, 34, 42]. Regarding the above, it is recommended to reduce of use of nitrate fertilizer in lands for prevention of methemoglobinemia, especially in the diet of children. On the other hand, due to the high supply of some products in the market due to low prices and high per capita consumption by people, especially in children, this is somewhat impossible. Therefore, it is necessary to have sufficient supervision on how to plant and harvest and import the products to be examined [16]. Considering that nitrate and nitrite levels in some of the foodstuffs studied are higher than the EU, it is recommended that control measures to reduce contamination and regular monitoring of products supplied by food and drug deputies of medical universities of the country should be considered promptly.

\section{Conclusion}

Since the various processes do not guarantee the absence of nitrogen compounds, the presence of this dangerous and highly toxic substance in vegetables is extremely important. Vegetables free of nitrogen compounds are desired for communities, but achieving this ideal is not easy. Accordingly, standards and rules have been set to reduce this substance in vegetables. Of course, the limits determined by the standard are widely variable and delicate scientific principles are involved in their regulation. It seems that the presence of the substance studied is related to nitrogen fertilizers and it is worth considering that in Iran there is almost no health control on the quality of vegetables. In order to achieve the desired quality, the cultivation conditions should be carefully monitored. Solutions to reduce nitrogen compounds should be considered, including the lack of nitrogen fertilizer and continuous and accurate monitoring of the handling and maintenance of vegetables by experienced experts. The prevalence of nitrates and nitrite in vegetables has imposed irreparable risks to human health. So, periodic and continuous control is vital. Although environmental and nutritional factors are an important parameter in the level of infection, the extent to which these factors are not well understood is not known.

\section{Acknowledgements}

The authors gratefully acknowledge the Research Council of Kermanshah University of Medical Sciences (Grant Number: 3003977) for its financial support.

\section{References}

1. SADEGHI E., HASHEMIAN A., SOLTANIAN M., SOLTANIAN S., MOHAMMADI M. Study on the effect of boiling and freezing process on nitrate and nitrite levels in abundant consumed vegetables. Iranian Journal of Nutrition Sciences \& Food Technology. 8, 201, 2013.

2. ALMASI A., DARGAHI A., HOSEINI AHAGH M. M., JANJANI H., MOHAMMADI M., TABANDEH L. Efficiency of a constructed wetland in controlling organic pollutants, nitrogen, and heavy metals from sewage. 
Journal of Chemical and Pharmaceutical Sciences. 9, 2924, 2016.

3. O'BRIEN J.A., VEGA A., BOUGUYON E., KROUK G., GOJON A., CORUZZI G., GUTIERREZ R.A. Nitrate transport, sensing, and responses in plants. Molecular plant. 9, 837, 2016.

4. SANTAMARIA P. Contributo degli ortaggi all'assunzione giornaliera di nitrato, nitrito e nitrosamine. Industrie alimentari. 36, 1329, 1997.

5. HORD N.G., TANG Y., BRYAN N.S. Food sources of nitrates and nitrites: the physiologic context for potential health benefits. The American journal of clinical nutrition. 90, 1, 2009.

6. THORUP-KRISTENSEN K. Are differences in root growth of nitrogen catch crops important for their ability to reduce soil nitrate-N content, and how can this be measured? Plant and Soil. 230, 185, 2001.

7. PAVLOU G.C., EHALIOTIS C.D., KAVVADIAS V.A. Effect of organic and inorganic fertilizers applied during successive crop seasons on growth and nitrate accumulation in lettuce. Scientia Horticulturae. 111, 319, 2007.

8. SADEGHI E., SHARAFI K., ALMASI A., DAYHIM M., AZIZI E., GHAYEBZADEH M. Study on the nitrite and nitrate levels changes by drying and frying processing in vegetables. Iranian Journal of Health and Environment. 7, 491, 2015.

9. SADEGHI E., HASHEMIAN A., SOLTANIAN M., SOLTANIAN S., MOHAMMADI M. Sadeghi, E., et al., Study of nitrite and nitrate levels in meat products distributed in Kermanshah. Iran Occupational Health. 11, 2014.

10. SADEGHI E., HASHEMIAN A., MOHAMMADI M., MOHAMMADI R. Study on the microbiological and chemical characterization of the meat product consumed in Kermanshah in 2012. Iranian Journal of Nutrition Sciences \& Food Technology. 7, 281, 2013.

11. HASHEMIMAJD K., FATHIACHACHILOI B. Estimation of nitrate dietary intake to food of Ardabil citizens. Agricultural Sci. 95, 91, 2008.

12. SPEIJERS G.J.A., VAN DEN BRANDT PA N.A.P., [ONLINE]., E.F.O.N.-N.C., AVAILABLE W.F.A.S., HTTP://WWW.INCHEM.ORG/DOCUMENTS/JECFA/ JECMONO/V50JE06. \& 2004]., H. J.

13. CROITOTU M.D., FULOP I., MIKLOS A., HOSSZU B., TATAR MUNTEAN A. Presence of nitrate and nitrite in vegetables grown for self-consumption. Farmacia 63, 115, 2015.

14. SUH J., PEAK O., KANG y., AHN J.E., JUNG J.S., An, Y.S., PARK., Lee S.J. Risk Assessment on Nitrate and Nitrite in Vegetables Available in Korean Diet. Journal of Applied Biological Chemistry. 56, 205, 2013.

15. ESTEGHAMATI A., GOUYA M. M., ABBASI M., DELAVARI A., ALIKHANI S., ALAEDINI F., SAFAIE A., FOROUZANFAR M., GREGG, E.W. Prevalence of diabetes and impaired fasting glucose in the adult population of Iran. Diabetes care. 31, 96, 2008.

16. TOP5OFANYTHING.COM.

17. PIRSAHEB M., PASDAR Y. Nitrate and nitrite in vegetables and summer crops consumed in Kermanshah (1389). Journal of Kermanshah University of Medical Sciences. 6, 76, 2011.

18. PIRSAHEB M., MORADI M. Evaluation of nitrate and nitrite vegetables and herbs grown in the southern and eastern plains of Kermanshah in 1390. Journal of Food Hygiene. 3, 41, 2014.
19. SHAHBAZZADEGAN S., SHAHBAZI B. Measuring the concentration of nitrate in fruit and vegetable supply in Ardabil. Journal of Ardabil University of Medical Sciences. 10, 38, 2010.

20. NOWROUZ P., TAGHIPOUR H., DASTGIRI S., BAFANDEH Y., HASHEMIMAJD K. Nitrate Determination of Vegetables in Varzeghan City, Northwestern Iran. Health promotion perspectives. 2, 244, 2012.

21. 2SADEGHI E., MOHAMMADI M., BOHLOLI OSKOII S., MESKINI H., MOHAMMADI R., ALMASI A. The effect of baking and freezing the vegetables consumed, nitrite and nitrate. International Journal of Food Industries. 8, 201, 2013.

22. ROSTAMZADE A., MOHAMMADI J. The resources and different nitrogen rates on nitrogen use efficiency and yield of cucumber green. Journal of Soil and Water. 23, 15, 2013.

23. REZAEI M., FANI A., MOINI A.L., MIRZAJANI P., MALEKIRAD A.A., RAFIEI M. Determining nitrate and nitrite content in beverages, fruits, vegetables, and stews marketed in Arak, Iran. International Scholarly Research Notices. 2014.

24. PIRSAHEB M., AHMADIAN M., RESHADAT S., POORMOHAMMADI A. The Measurement of Nitrite and Nitrate Contents of Tomato from the Vast Plain of Kermanshah State of Iran. International Journal of Agriculture and Crop Sciences. 7, 1553, 2014.

25. EFTEKHARI M.H. Accumulation of nitrate and nitrite in a pile of spinach (Spinacia oleracea L.) native to Iran. Journal of Plant Production. 37, 111, 2014.

26. LAHIJI A.A., BASHARDOOST L. Evaluation of nitrate accumulation in Hamedan potato farms.

27. SHARIATI-RAD M., IRANDOUST M., NIAZI, F. Determination of nitrite in food samples by second-order calibration of kinetic spectrophotometric data. Journal of analytical chemistry. 71, 660, 2016.

28. FAZLZADEH M., SERAJAMANI R., ROSTAMY R., REZAEI M., SHAHRIARI R., SADEGHI H. Comparing the Effect of Conventional Inorganic and Humic Fertilizers' Use on Nitrate Levels of Potatoes: A Case Study of Agricultural Lands in Ardabil City. Journal of Health. 8, 416, 2017.

29. MOUSAVI MOAYEID F., CHERAGHI M., LORESTANI B. Investigation of the Amount of Phosphate and Nitrate Accumulation in Consumable Onion in Hamedan City. J Neyshabur Univ Med Sci. 4, 82, 2017.

30. JALINI M., DOOSTI F. Evaluation of nitrate accumulation in potato and tomato. Journal of Environmen. 50, 62, 2011.

31. SHOKRZADEH M., SHOKRAVIE M., EBADI A., BABAEE Z., TARIGHATI A. The measurement of nitrate and nitrite content in leek and spinach sampled from central cities of Mazandaran State of Iran. World Applied Sciences Journal. 2, 121, 2007.

32. SHAHLAEI A., ANSARI N.A., DEHKORDIE F.S. Evaluation of nitrate and nitrite content of Iran Southern (Ahwaz) vegetables during winter and spring of 2006. Asian J. Plant Sci. 6, 1197, 2007.

33. ROUSTAA M.J., LOTFIB E., SHAMSALAMC N., MOUSAVID F., SOLEIMAN L. Nitrate Situation in Some Vegetables and the Necessity of Crop Production via Organic Farming. in $19^{\text {th }}$ World Congress of Soil Science. 2010.

34. AFALI S., ELAHI R. Measuring nitrate and nitrite concentrations in vegetables, fruits in Shiraz. Journal of Applied Sciences and Environmental Management. 18, 451, 2014. 
35. KESHAVARZ M., MAZLOOMI S.M., BABAJAFARI S. The Effect of Home Cooking Method and Refrigeration Processes on the Level of Nitrate and Nitrite In Spinach. Journal of health sciences and surveillance system. 3, 88, 2015.

36. GHOLAMI A., KELEY K., KELEY M.A. Studying of the Nitrate Contamination in Garden Cress Plant in Dezful, Khouzestan, Iran. International Journal of Scientific Research in Knowledge. 1, 93, 2013.

37. ARDEKANI SOBHAN S., OFIONI M., SOFIANI MAHBOBI N. The concentration of nitrate in some herbal products in Esfahan. Journal of Ecology. 31, 69, 2005.

38. KAFESHANI O., YAHAI M., ENTEZARI M.H., HASSANZADEH A., MOHEBAT L., TORABI A. Compare the amount of nitrate in vegetables irrigated with river water and well water. Journal of Preventive Medicine. 9, 2196, 2013.

39. RAHMANI H.R. Soil and Water Research Division of Agriculture and Natural Resources Research Center, Check the status of nitrate in the soil, water and plants, too, the land area of vegetable growers in Baraan Esfahan. Journal of Preventive Medicine. 9, 196, 2013.

40. SAEEDIFAR F., ZIARATI P., RAMEZAN Y. Nitrate and Heavy Metal Contents in Eggplant (Solanum melongena) cultivated in the farmlands in the south of Tehran-Iran. International Journal of Farming and Allied Sciences. 3, 60, 2014.

41. BAHADORAN Z., MIRMIRAN P., JEDDI S., AZIZI F., GHASEMI A., HADAEGH, F. Nitrate and nitrite content of vegetables, fruits, grains, legumes, dairy products, meats and processed meats. Journal of Food Composition and Analysis. 51, 93, 2016.

42. PORMOGHIM M., SADEGHI MAKI A., KOMAILI R., GOLESTAN B., PIRALI M. Determination of nitrate contents of lettuce, tomato and potato Released Right vegetables Tehran by HPLC. Journal of Nutrition and Food Technology. 1, 63, 2010.

43. NUNEZ GONZALE M.T., OSBURN WHARDIN M., LONGNECKER M., GARG H., BRYAN N., KEETON J. Survey of Nitrate and Nitrite Concentrations in Conventional and Organic-Labeled Raw Vegetables at Retail. Journal of Food Science. 80, 111, 2015.

44. IAMMARINO M., Di TARANTO A., CRISTINO M. Monitoring of nitrites and nitrates levels in leafy vegetables (spinach and lettuce): a contribution to risk assessment. Journal of the Science of Food and Agriculture. 94, 773, 2014.

45. HABERMEYER M., ROTH A., GUTH S., DIEL P., ENGEL K. H., EPE B., FURST P., HEINZ V., HUMPF H. U., JOOST H. G. Nitrate and nitrite in the diet: how to assess their benefit and risk for human health. Molecular nutrition \& food research. 59, 106, 2015.

46. ALAMIAN M., HEIDARI M., ALAMZADE ANSARI N. Evaluation of nitrate concentration and nitrate reductase activity in various stages of growth in Iranian masses spinach (Spinacia oleracea L). Journal of Plant Production. 34, 89, 2014. 